\title{
Evolutionary Quasi-Variational-Hemivariational Inequalities I: Existence and Optimal Control
}

\author{
Shengda Zeng ${ }^{1,2} \cdot$ Dumitru Motreanu ${ }^{3,4} \cdot$ Akhtar A. Khan $^{5}$
}

Received: 30 April 2021 / Accepted: 15 October 2021 / Published online: 2 November 2021

(c) The Author(s) 2021

\begin{abstract}
We study a nonlinear evolutionary quasi-variational-hemivariational inequality (in short, (QVHVI)) involving a set-valued pseudo-monotone map. The central idea of our approach consists of introducing a parametric variational problem that defines a variational selection associated with (QVHVI). We prove the solvability of the parametric variational problem by employing a surjectivity theorem for the sum of operators, combined with Minty's formulation and techniques from the nonsmooth analysis. Then, an existence theorem for (QVHVI) is established by using Kluge's fixed point theorem for set-valued operators. As an application, an abstract optimal control problem for the (QVHVI) is investigated. We prove the existence of solutions for the optimal control problem and the weak sequential compactness of the solution set via the Weierstrass minimization theorem and the Kuratowski-type continuity properties.
\end{abstract}

Keywords Evolutionary quasi-variational-hemivariational inequality · surjectivity theorems · Kuratowski upper limit · optimal control.

Mathematics Subject Classification 47J20 - 58Exx · 35Kxx · 34H05 - 49J52 - 74B20

\section{Introduction}

Variational inequalities constitute one of the most vibrant branches of applied mathematics that have been extended in a multitude of directions. One of the notable generalizations of variational inequalities that have attracted a great deal of attention is the so-called hemivariational inequality, pioneered in the 1980s by P.D. Panagiotopoulos $[39,40]$. During the last several decades, hemivariational inequalities

Communicated by Qamrul Hasan Ansari.

Dedicated to Professor Franco Giannessi on the occasion of his 85th birthday.

Extended author information available on the last page of the article 
have been explored extensively in novel engineering applications. In contrast to variational inequalities, which emerge from convex energy principles, the hemivariational inequalities are connected to nonsmooth and nonconvex energy functionals and naturally involve generalized derivatives of nonsmooth functionals, see [5]. For some of the recent developments in hemivariational inequalities and their applications, we refer the reader to $[1,7,8,11,15-17,26-30,38,41-44]$, and the cited references. The necessity to understand time-dependent applied models involving nonsmooth and nonconvex energy principles gives rise to evolutionary hemivariational inequalities. A prototypical example is the study of nonstationary fluid flow problems modeled by nonmonotone and set-valued frictional laws, see [34-36].

In this paper, our focus is on a new class of evolutionary quasi-variationalhemivariational inequalities (in short, (QVHVI)), where the underlying constraint set depends on the unknown solution. In contrast to the classical variational and hemivariational inequalities where the constraints sets remain fixed, their quasi-variants pose novel theoretical and computational challenges due to the peculiar dependence on the unknown solution. On the other hand, since the quasi-variants appear very frequently in applied models, they have received significant attention in recent years, see $[9,13,14,18,20-24,27,31-33]$ and the cited references therein.

This paper is devoted to a thorough study of an evolutionary quasi-variationalhemivariational inequality described in Sect. 3. Besides some auxiliary results, we give two novel results. The first result of this paper proves the solvability and weak sequential compactness of the solution set for the (QVHVI) given in Problem 1. In proving this result, the central role is played by Kluge's fixed point theorem, Minty's formulation, and some techniques from the nonsmooth analysis. Our second result addresses an optimal control problem associated with the above (QVHVI). Here, our approach is based on using minimizing sequences in conjunction with the Kuratowskitype continuity properties.

We organize the contents of this paper into four sections. Section 2 presents the necessary background material. In Sect. 3, we investigate the existence and compactness of the solution set of (QVHVI) in Problem 1. Section 4 is devoted to a nonlinear optimal control problem associated with (QVHVI). The paper concludes with some remarks.

\section{Mathematical Background}

We start with a brief discussion on some classes of set-valued operators of monotone type. An elaborate presentation can be found in $[4,37,45]$.

Definition 1 Let $E$ be a reflexive Banach space with the dual $E^{*}$ and let $A: D(A) \subset$ $E \rightarrow 2^{E^{*}}$ be a set-valued mapping with the domain $D(A)=\{u \in E \mid A u \neq \emptyset\}$. We say that

(i) $A$ is monotone, if

$$
\left\langle u^{*}-v^{*}, u-v\right\rangle_{E} \geq 0 \text { for all } u^{*} \in A u, v^{*} \in A v \text { and } u, v \in D(A)
$$


(ii) $A$ is maximal monotone, if it is monotone and has a maximal graph, that is,

$$
\left\langle u^{*}-v^{*}, u-v\right\rangle_{E} \geq 0 \text { for all } u^{*} \in A u \text { and } u \in D(A)
$$

implies $v \in D(A)$ and $v^{*} \in A v$;

(iii) $A$ is pseudomonotone with respect to $D(\mathcal{L})$ (or $\mathcal{L}$-pseudomonotone) for a linear, closed, densely defined, and maximal monotone operator $\mathcal{L}: D(\mathcal{L}) \subset E \rightarrow E^{*}$ if

(a) for each $u \in E$, the set $A u$ is nonempty, bounded, closed, and convex in $E^{*}$;

(b) $A$ is upper semicontinuous from any finite-dimensional subspace of $E$ to $E^{*}$ endowed with the weak topology;

(c) for any sequences $\left\{u_{n}\right\} \subset D(\mathcal{L})$ and $\left\{u_{n}^{*}\right\} \subset E^{*}$ with

$$
\left\{\begin{array}{l}
u_{n} \rightarrow u \text { weakly in } E \\
\mathcal{L} u_{n} \rightarrow \mathcal{L} u \text { weakly in } E^{*}, \\
u_{n}^{*} \in A u_{n} \text { for all } n \in \mathbb{N} \\
u_{n}^{*} \rightarrow u^{*} \text { weakly in } E^{*} \\
\limsup _{n \rightarrow \infty}\left\langle u_{n}^{*}, u_{n}-u\right\rangle_{E} \leq 0
\end{array}\right.
$$

we have $u^{*} \in A u$ and $\lim _{n \rightarrow \infty}\left\langle u_{n}^{*}, u_{n}\right\rangle_{E}=\left\langle u^{*}, u\right\rangle_{E}$.

The sum of set-valued pseudomonotone operators is pseudomonotone (see, e.g., [4, Theorem 2.124]). The same holds for pseudomonotone operators with respect to $D(\mathcal{L})$.

Proposition 2 Assume that $A: E \rightarrow 2^{E^{*}}$ and $B: E \rightarrow 2^{E^{*}}$ are pseudomonotone with respect to $D(\mathcal{L})$ and bounded in the sense of mapping bounded sets to bounded sets. Then, the set-valued sum $A+B: E \rightarrow 2^{E^{*}}$ is pseudomonotone with respect to $D(\mathcal{L})$.

Proof Since the sets $A u$ and $B u$ are nonempty, bounded, closed, and convex in $E^{*}$, the same remains true for $(A+B) u=A u+B u$. The values of $A$ and $B$ being compact sets in the weak topology of $E^{*}$ permit the use of [19, Theorem 1.2.14] to guarantee that $A+B$ is upper semicontinuous from any finite-dimensional subspace of $E$ to $E^{*}$ endowed with the weak topology.

Let the sequences $\left\{u_{n}\right\} \subset D(\mathcal{L}),\left\{u_{n}^{*}\right\} \subset E^{*}$ and $\left\{v_{n}^{*}\right\} \subset E^{*}$ satisfy $u_{n} \rightarrow u$ weakly in $E, \mathcal{L} u_{n} \rightarrow \mathcal{L} u$ weakly in $E^{*}, u_{n}^{*} \in A u_{n}$ and $v_{n}^{*} \in B u_{n}$ for all $n \in \mathbb{N}$, $u_{n}^{*}+v_{n}^{*} \rightarrow w^{*}$ weakly in $E^{*}$, and $\lim _{\sup _{n \rightarrow \infty}}\left\langle u_{n}^{*}+v_{n}^{*}, u_{n}-u\right\rangle_{E} \leq 0$. We claim that $\limsup _{n \rightarrow \infty}\left\langle u_{n}^{*}, u_{n}-u\right\rangle_{E} \leq 0$ and $\limsup _{n \rightarrow \infty}\left\langle v_{n}^{*}, u_{n}-u\right\rangle_{E} \leq 0$. Arguing by contradiction, along a subsequence, we suppose

$$
\limsup _{n \rightarrow \infty}\left\langle u_{n}^{*}, u_{n}-u\right\rangle_{E}=\lim _{k \rightarrow \infty}\left\langle u_{n_{k}}^{*}, u_{n_{k}}-u\right\rangle_{E}=: a>0
$$

Then, the inequality $\lim \sup \left\langle v_{n_{k}}^{*}, u_{n_{k}}-u\right\rangle_{E} \leq-a$ is true. Consequently, it follows that $\lim _{n \rightarrow \infty}\left\langle v_{n_{k}}^{*}, u_{n_{k}}\right\rangle_{E}=\left\langle v^{*}, u\right\rangle_{E}$ because $B: E \rightarrow 2^{E^{*}}$ is pseudomonotone with 
respect to $D(\mathcal{L})$. The preceding inequality leads to the contradiction $0 \leq-a$, which proves the claim.

Using that $A: E \rightarrow 2^{E^{*}}$ and $B: E \rightarrow 2^{E^{*}}$ are bounded and the space $E^{*}$ is reflexive, up to subsequences, it holds that $u_{n}^{*} \rightarrow u^{*}$ weakly in $E^{*}$ and $v_{n}^{*} \rightarrow v^{*}$ weakly in $E^{*}$ for $u^{*}, v^{*} \in E^{*}$ with $u^{*}+v^{*}=w^{*}$. Then, the pseudomonotonicity of $A$ and $B$ entails $u^{*} \in A u, v^{*} \in B u, \lim _{n \rightarrow \infty}\left\langle u_{n}^{*}, u_{n}\right\rangle_{E^{*} \times E}=\left\langle u^{*}, u\right\rangle_{E}$ and $\lim _{n \rightarrow \infty}\left\langle v_{n}^{*}, u_{n}\right\rangle_{E}=$ $\left\langle v^{*}, u\right\rangle_{E}$. It turns out that $w^{*} \in A u+B u$ and $\lim _{n \rightarrow \infty}\left\langle u_{n}^{*}+v_{n}^{*}, u_{n}\right\rangle_{E}=\left\langle w^{*}, u\right\rangle_{E}$, thus completing the proof.

Next, we focus on some tools from convex analysis and nonsmooth analysis. Let $E$ be a Banach space with its dual $E^{*}$ and let $\varphi: E \rightarrow \mathbb{R} \cup\{+\infty\}$ be a proper, convex, and lower semicontinuous function. The subdifferential of $\varphi$ is the mapping $\partial_{C} \varphi: E \rightarrow 2^{E^{*}}$ defined by

$$
\partial_{C} \varphi(u)=\left\{u^{*} \in E^{*} \mid\left\langle u^{*}, v-u\right\rangle_{E} \leq \varphi(v)-\varphi(u) \text { for all } v \in E\right\} .
$$

We recall the following basic result (see, e.g., [6, Theorem 6.3.19]).

Theorem 3 Let $E$ be a real Banach space and $\varphi: E \rightarrow \mathbb{R} \cup\{+\infty\}$ be a proper, convex, and lower semicontinuous function. Then, $\partial_{C} \varphi: E \rightarrow 2^{E^{*}}$ is a maximal monotone operator.

Let $J: E \rightarrow \mathbb{R}$ be a locally Lipschitz function and $u, v \in E$. The generalized directional derivative of $J$ at $u$ in the direction $v$ is defined as

$$
J^{0}(u ; v):=\limsup _{w \rightarrow u, t \downarrow 0} \frac{J(w+t v)-J(w)}{t},
$$

and the generalized gradient $\partial J: E \rightarrow 2^{E^{*}}$ of $J: E \rightarrow \mathbb{R}$ is defined by

$$
\partial J(u)=\left\{\xi \in E^{*} \mid J^{0}(u ; v) \geq\langle\xi, v\rangle_{E} \text { for all } v \in E\right\} .
$$

The following result summarizes some basic results on generalized gradients, (see [37, Proposition 3.23]).

Proposition 4 Let $J: E \rightarrow \mathbb{R}$ be locally Lipschitz with constant $L_{u}>0$ near $u \in E$. Then, we have:

(a) the function $v \mapsto J^{0}(u ; v)$ is positively homogeneous, is subadditive, and satisfies

$$
\left|J^{0}(u ; v)\right| \leq L_{u}\|v\|_{E} \text { for all } v \in E
$$

(b) $(u, v) \mapsto J^{0}(u ; v)$ is upper semicontinuous;

(c) $\partial J(u)$ is a nonempty, convex, and weakly* compact subset of $E^{*}$ with

$$
\|\xi\|_{E^{*}} \leq L_{u} \text { for all } \xi \in \partial J(u)
$$

(d) for all $v \in E, J^{0}(u ; v)=\max \left\{\langle\xi, v\rangle_{E} \mid \xi \in \partial J(u)\right\}$. 
Besides, we mention the concept of strongly quasi-boundedness for set-valued operators, see [10, Definition 2.14].

Definition 5 Let $E$ be a reflexive Banach space with the dual $E^{*}$. A set-valued map $A: D(A) \subset E \rightarrow 2^{E^{*}}$ is called strongly quasi-bounded if for each $M>0$ there exists $K_{M}>0$ such that for any $u \in D(A)$ and $u^{*} \in A u$ with

$$
\left\langle u^{*}, u\right\rangle_{E} \leq M \text { and }\|u\|_{E} \leq M,
$$

it follows that $\left\|u^{*}\right\|_{E^{*}} \leq K_{M}$.

The following condition ensuring strongly quasi-boundedness was given by Browder-Hess [3, Proposition 14].

Proposition 6 Let $E$ be a reflexive Banach space with its dual $E^{*}$. If $A: D(A) \subset E \rightarrow$ $2^{E^{*}}$ is a monotone operator such that $0 \in \operatorname{int} D(A)$, then $A$ is strongly quasi-bounded.

We also recall from [10, Theorem 3.1] the following surjectivity result:

Theorem 7 Let $E$ be a reflexive, strictly convex Banach space, let $\mathcal{L}: D(\mathcal{L}) \subset E \rightarrow$ $E^{*}$ be a linear, closed, densely defined, and maximal monotone operator, and let $A: E \rightarrow 2^{E^{*}}$ be a bounded and $\mathcal{L}$-pseudomonotone operator such that

$$
\langle A u, u\rangle_{E} \geq r\left(\|u\|_{E}\right)\|u\|_{E} \text { for all } u \in E,
$$

where $r: \mathbb{R}_{+} \rightarrow \mathbb{R}$ satisfies $r(s) \rightarrow+\infty$ as $s \rightarrow+\infty$. If $B: D(B) \subset E \rightarrow 2^{E^{*}}$ is a maximal monotone operator which is strongly quasi-bounded and $0 \in B(0)$, then $\mathcal{L}+A+B$ is surjective, that is, its range is $E^{*}$.

We conclude this section by recalling Kluge's fixed point theorem [25].

Theorem 8 Let $C$ be a nonempty, closed, and convex subset of a reflexive Banach space $Z$. Assume that $\Psi: C \rightarrow 2^{C}$ is a set-valued map such that for every $u \in C$ the set $\Psi(u)$ is nonempty, closed, and convex, and the graph of $\Psi$ is sequentially weakly closed. If $\Psi(C)$ is bounded, then $\Psi$ has a fixed point.

\section{Existence Results}

Let $\mathcal{V}$ and $\mathcal{E}$ be reflexive Banach spaces with duals $\mathcal{V}^{*}$ and $\mathcal{E}^{*}$, let $\mathcal{X}$ be a nonempty subset of $\mathcal{V}$, and let $\gamma: \mathcal{V} \rightarrow \mathcal{E}$ be a bounded linear operator. Let $\langle\cdot, \cdot\rangle_{\mathcal{V}}$ be the duality pairing of $\mathcal{V}^{*}$ and $\mathcal{V}$ and let $\langle\cdot, \cdot\rangle_{\mathcal{E}}$ be the duality pairing of $\mathcal{E}^{*}$ and $\mathcal{E}$. Given a linear, closed, densely defined, and maximal monotone operator $\mathcal{L}: D(\mathcal{L}) \rightarrow \mathcal{V}^{*}$, two set-valued operators $\mathcal{T}: \mathcal{V} \rightarrow 2^{\mathcal{V}^{*}}$ and $\mathcal{K}: \mathcal{X} \rightarrow 2^{\mathcal{X}}$, a proper, convex, and lower semicontinuous function $\varphi: \mathcal{V} \rightarrow \mathbb{R} \cup\{+\infty\}$, a locally Lipschitz function $J: \mathcal{E} \rightarrow \mathbb{R}$, and an element $f \in \mathcal{V}^{*}$, we formulate the following evolutionary problem in the form of (QVHVI):

Problem 1 Find $u \in \mathcal{K}(u) \cap D(\mathcal{L})$ such that for some $u^{*} \in \mathcal{T}(u)$, we have $\left\langle\mathcal{L} u+u^{*}-f, v-u\right\rangle_{\mathcal{V}}+J^{0}(\gamma u ; \gamma(v-u))+\varphi(v)-\varphi(u) \geq 0, \quad$ for all $v \in \mathcal{K}(u)$, 
where $J^{0}$ is the generalized derivative of $J$ to be defined shortly.

We now formulate the necessary assumptions on the data of (QVHVI):

$H(0): \mathcal{V}$ is a reflexive, strictly convex Banach space, $\mathcal{E}$ is a reflexive Banach space, $\overline{\text { and } \mathcal{X}}$ is a nonempty, closed, and convex subset of $\mathcal{V}$.

$H(\mathcal{L}): \mathcal{L}: D(\mathcal{L}) \subset \mathcal{V} \rightarrow \mathcal{V}^{*}$ is a linear, closed, densely defined, and maximal monotone operator.

$H(\mathcal{T}): \mathcal{T}: \mathcal{V} \rightarrow 2^{\mathcal{V}^{*}}$ is bounded and pseudomonotone and there exist constants $m_{\mathcal{T}}>$ $\overline{0, d_{\mathcal{T}}} \geq 0$ and $p>1$ such that

$$
\inf _{u^{*} \in \mathcal{T} u}\left\langle u^{*}, u\right\rangle_{\mathcal{V}} \geq m_{\mathcal{T}}\|u\|_{\mathcal{V}}^{p}-d_{\mathcal{T}}, \quad \text { for all } u \in \mathcal{V}
$$

$H(\gamma): \gamma: \mathcal{V} \rightarrow \mathcal{E}$ is a linear and compact operator.

$\overline{H(J)}: J: \mathcal{E} \rightarrow \mathbb{R}$ is a locally Lipschitz function for which there exist constants $p>1$, $\overline{\theta \in[1}, p]$ and $c_{J}>0$ with $m_{\mathcal{T}} \kappa(\theta)>c_{J}\|\gamma\|^{p}$ and

$$
\|\xi\|_{\mathcal{E}^{*}} \leq c_{J}\left(1+\|z\|_{\mathcal{E}}^{\theta-1}\right)
$$

for all $\xi \in \partial J(u)$ and $u \in \mathcal{E}$, where

$$
\kappa(\theta):=\left\{\begin{array}{ll}
1 & \text { if } \theta=p \\
+\infty & \text { if } \theta<p
\end{array} .\right.
$$

$H(\mathcal{K}): \mathcal{K}: \mathcal{X} \rightarrow 2^{\mathcal{X}}$ is such that

(i) For each $w \in \mathcal{X}$ the set $\mathcal{K}(w)$ is nonempty, closed, and convex in $\mathcal{V}$.

(ii) $0 \in \operatorname{int} \bigcap_{w \in \mathcal{X}} \mathcal{K}(w)$.

(iii) If the sequences $\left\{w_{n}\right\} \subset \mathcal{X}$ and $\left\{u_{n}\right\} \subset \mathcal{K}\left(w_{n}\right) \cap D(\mathcal{L})$ satisfy

$w_{n} \rightarrow w$ weakly in $\mathcal{V}, u_{n} \rightarrow u$ weakly in $\mathcal{V}$ and $\mathcal{L} u_{n} \rightarrow \mathcal{L} u$ weakly in $\mathcal{V}^{*}$

for some $w \in \mathcal{X}$ and $u \in D(\mathcal{L})$, then $u \in \mathcal{K}(w)$.

(iv) For every sequence $\left\{w_{n}\right\} \subset \mathcal{X}$ with $w_{n} \rightarrow w$ weakly in $\mathcal{V}$ and for every $v \in$ $\mathcal{K}(w)$, there exist a subsequence $\left\{w_{n_{k}}\right\}$ of $\left\{w_{n}\right\}$ and a sequence $\left\{v_{n_{k}}\right\} \subset \mathcal{X}$ with $v_{n_{k}} \in \mathcal{K}\left(w_{n_{k}}\right)$ such that $v_{n_{k}} \rightarrow v$ in $\mathcal{V}$ as $k \rightarrow \infty$.

$H(\varphi): \varphi: \mathcal{V} \rightarrow \mathbb{R} \cup\{+\infty\}$ is a proper, convex, and lower semicontinuous function $\overline{\text { satisfying }}$

$$
\bigcup_{w \in \mathcal{X}} \mathcal{K}(w) \subset \operatorname{int} D(\varphi), \quad 0 \in \operatorname{int} D(\varphi), \quad 0 \in \partial_{C} \varphi(0) .
$$

A fruitful approach for quasi-variational inequalities is defining a variational selection and finding its fixed point. To elucidate, we first consider, each $w \in \mathcal{X}$, the following parametric problem: 
Problem 2 Find $u \in \mathcal{K}(w) \cap D(\mathcal{L})$ such that for some $u^{*} \in \mathcal{T}(u)$, we have

$$
\begin{aligned}
& \left\langle\mathcal{L} u+u^{*}-f, v-u\right\rangle_{\mathcal{V}}+J^{0}(\gamma u ; \gamma(v-u)) \\
& +\varphi(v)-\varphi(u) \geq 0, \quad \text { for all } v \in \mathcal{K}(w) .
\end{aligned}
$$

This permits us to define the set-valued mapping $\mathcal{S}: \mathcal{X} \rightarrow 2^{\mathcal{X}}$ that assigns to each $w \in \mathcal{X}$ the solution set $\mathcal{S}(w)$ of Problem 2 corresponding to $w$. This set-valued map is the so-called variational selection for the (QVHVI) defined in Problem 1. Evidently, any fixed of $\mathcal{S}$ is a solution of Problem 1.

We will need the following technical result that circumvents difficulties related to the unboundedness of $\mathcal{L}$.

Lemma 9 Assume that $H(0), H(\mathcal{L}), H(\mathcal{T}), H(\gamma), H(J), H(\varphi)$, and $H(\mathcal{K})(\mathrm{i})$-(ii) hold. If $\left\{w_{n}\right\} \subset \mathcal{X}$ and $\left\{u_{n}\right\} \subset D(\mathcal{L})$ are sequences such that $\left\{u_{n}\right\}$ is bounded in $\mathcal{V}$ and $u_{n} \in \mathcal{S}\left(w_{n}\right)$ for each $n \in \mathbb{N}$, then $\left\{\mathcal{L} u_{n}\right\}$ is bounded in $\mathcal{V}^{*}$.

Proof By assumption, there exists $u_{n} \in \mathcal{K}\left(w_{n}\right)$ such that for some $u_{n}^{*} \in \mathcal{T} u_{n}$, we have $\left\langle\mathcal{L} u_{n}+u_{n}^{*}-f, v-u_{n}\right\rangle \mathcal{V}+J^{0}\left(\gamma u_{n} ; \gamma\left(v-u_{n}\right)\right)+\varphi(v)-\varphi\left(u_{n}\right) \geq 0$ for all $v \in \mathcal{K}\left(w_{n}\right)$.

By hypothesis $H(\mathcal{K})(\mathrm{ii})$, there is an arbitrarily small neighborhood $C$ of 0 in $\mathcal{V}$ satisfying $C \subset \mathcal{K}\left(w_{n}\right)$ for all $n \in \mathbb{N}$. We will show that

$$
\inf _{n \in \mathbb{N}, v \in C}\left\langle\mathcal{L} u_{n}, v\right\rangle \mathcal{V}>-\infty
$$

which proves our result (see similar ideas in part (I) of the proof of [45, Proposition 32.33] and in Step 4 of the proof of [20, Theorem 5.1]). Inequality (2) and the monotonicity of $\mathcal{L}$ yield

$\left\langle\mathcal{L} u_{n}, v\right\rangle_{\mathcal{V}} \geq\left\langle u_{n}^{*}-f, u_{n}-v\right\rangle_{\mathcal{V}}-J^{0}\left(\gamma u_{n} ; \gamma\left(v-u_{n}\right)\right)+\varphi\left(u_{n}\right)-\varphi(v)$ for all $v \in C$

Thanks to hypothesis $H(\mathcal{T})$ and the fact that $\left\{u_{n}\right\}$ is bounded in $\mathcal{V}$, the sequence $\left\{u_{n}^{*}\right\}$ is bounded in $\mathcal{V}^{*}$. We deduce from the boundedness of $\left\{u_{n}\right\}$ and $C$, hypothesis $H(J)$ and Proposition 4 that $J^{0}\left(\gamma u_{n} ; \gamma\left(v-u_{n}\right)\right)$ is bounded. By condition $H(\varphi)$, it follows that $\varphi$ is bounded from below by an affine function (see, e.g., [2, Proposition 1.10]). Furthermore, $\varphi$ is bounded on $C$ because $C$ can be chosen arbitrarily small in int $D(\varphi)$. We conclude that

$$
\inf _{n \in \mathbb{N}, v \in C}\left[\left\langle u_{n}^{*}-f, u_{n}-v\right\rangle_{\mathcal{V}}-J^{0}\left(\gamma u_{n} ; \gamma\left(v-u_{n}\right)\right)+\varphi\left(u_{n}\right)-\varphi(v)\right]>-\infty,
$$

whence (3) follows via (4), which completes the proof.

The following result provides useful information on the set-valued mapping $\mathcal{S}$.

Lemma 10 Assume that $H(0), H(\mathcal{L}), H(\mathcal{T}), H(\gamma), H(J), H(\varphi)$, and $H(\mathcal{K})(\mathrm{i})-(\mathrm{ii})$ remain valid. Then, the following statements hold: 
(i) $\mathcal{S}(w) \neq \emptyset$, for all $w \in \mathcal{X}$.

(ii) The set $\mathcal{S}(w)$ is closed for all $w \in \mathcal{X}$.

(iii) The set $\mathcal{S}(\mathcal{X})$ is bounded in $\mathcal{V}$.

Proof (i). Fix $w \in \mathcal{X}$ and consider the function $\Phi: \mathcal{V} \rightarrow \mathbb{R} \cup\{+\infty\}$ defined by

$$
\Phi(u)= \begin{cases}\varphi(u) & \text { if } u \in \mathcal{K}(w), \\ +\infty & \text { otherwise }\end{cases}
$$

Problem 2 reads as follows: Find $u \in \mathcal{K}(w) \cap D(\mathcal{L})$ such that for some $u^{*} \in \mathcal{T}(u)$, we have

$\left\langle\mathcal{L} u+u^{*}-f, v-u\right\rangle_{\mathcal{V}}+J^{0}(\gamma u ; \gamma(v-u))+\Phi(v)-\Phi(u) \geq 0, \quad$ for all $\left.v \in \mathcal{X} 5\right)$

For the solvability of (5), we reformulate it as the inclusion problem of finding $u \in \mathcal{K}(w) \cap D(\mathcal{L})$ such that

$$
f \in \mathcal{L} u+\mathcal{T} u+\gamma^{*} \partial J(\gamma u)+\partial_{C} \Phi(u)
$$

Let $\mathcal{F}: \mathcal{V} \rightarrow 2^{\mathcal{V}^{*}}$ be defined by

$$
\mathcal{F}(u):=\mathcal{T} u+\gamma^{*} \partial J(\gamma u) \text { for all } u \in \mathcal{V}
$$

The boundedness of $\mathcal{T}$ and hypotheses $H(J)$ and $H(\gamma)$ confirm that $\mathcal{F}: \mathcal{V} \rightarrow 2^{\mathcal{V}^{*}}$ is bounded.

Next, we claim that $\mathcal{F}$ is pseudomonotone with respect to $D(\mathcal{L})$. First, we prove that $u \mapsto \gamma^{*} \partial J(\gamma u)$ is strongly-weakly upper semicontinuous. Let $B$ be a weakly closed set in $\mathcal{V}^{*}$ and let $u_{n} \rightarrow u$ in $\mathcal{V}$ as $n \rightarrow \infty$ so that for every $n \in \mathbb{N}$, there exists $\xi_{n} \in \partial J\left(\gamma u_{n}\right)$ satisfying $\gamma^{*} \xi_{n} \in \gamma^{*} \partial J\left(\gamma u_{n}\right) \cap B$. The boundedness of $\partial J$ implies that the sequence $\left\{\xi_{n}\right\}$ is bounded in $\mathcal{E}^{*}$. We may assume that $\xi_{n} \rightarrow \xi$ weakly in $\mathcal{E}^{*}$ for some $\xi \in \mathcal{E}^{*}$. Because of the weak closedness of $B$ and linearity of $\gamma^{*}$, we have $\gamma^{*} \xi \in B$. For each $n \in \mathbb{N}$, we have

$$
\left\langle\xi_{n}, \gamma v\right\rangle_{\mathcal{E}} \leq J^{0}\left(\gamma u_{n} ; \gamma v\right) \text { for all } v \in \mathcal{V}
$$

By the upper semicontinuity of $J^{0}$ and compactness of $\gamma$, we get

$$
\langle\xi, \gamma v\rangle_{\mathcal{E}}=\lim _{n \rightarrow \infty}\left\langle\xi_{n}, \gamma v\right\rangle_{\mathcal{E}} \leq \limsup _{n \rightarrow \infty} J^{0}\left(\gamma u_{n} ; \gamma v\right) \leq J^{0}(\gamma u ; \gamma v) \text { for all } v \in \mathcal{V},
$$

implying that $\gamma^{*} \xi \in \gamma^{*} \partial J(\gamma u) \cap B$ (see, e.g., [37, Proposition 3.8]).

Now, let $u_{n} \rightarrow u$ weakly in $\mathcal{V}$ and $u_{n}^{*}=\gamma^{*} \xi_{n} \rightarrow u^{*}$ weakly in $\mathcal{V}^{*}$ with $\xi_{n} \in$ $\partial J\left(\gamma u_{n}\right)$ for every $n \in \mathbb{N}$. The compactness of $\gamma: \mathcal{V} \rightarrow \mathcal{E}$ implies $\gamma u_{n} \rightarrow \gamma u$ strongly in $\mathcal{E}$. The boundedness of $\partial J$ ensures along a relabeled subsequence $\xi_{n} \rightarrow \xi$ weakly in $\mathcal{E}^{*}$ for some $\xi \in \mathcal{E}^{*}$. From the strong-weak closedness of the graph of $\partial J$, we infer that $\xi \in \partial J(\gamma u)$, thus $\gamma^{*} \xi \in \gamma^{*} \partial J(\gamma u)$, and $\lim _{n \rightarrow \infty}\left\langle u_{n}^{*}, u_{n}\right\rangle_{\mathcal{V}}=\left\langle u^{*}, u\right\rangle_{\mathcal{V}}$ since $\gamma$ is compact. It turns out that $u \mapsto \gamma^{*} \partial J(\gamma u)$ is pseudomonotone, so pseudomonotone 
with respect to $D(\mathcal{L})$. Combining with hypothesis $H(\mathcal{T})$, we can apply Proposition 2 to deduce that $\mathcal{F}$ is pseudomonotone with respect to $D(\mathcal{L})$.

The next step is to prove that $\mathcal{F}$ is coercive. For this, $H(\mathcal{T})$ and $H(J)$ ensure that for any $u \in \mathcal{V}$, we have

$$
\begin{aligned}
\langle\mathcal{F} u, u\rangle_{\mathcal{V}} & \geq m_{\mathcal{T}}\|u\|_{\mathcal{V}}^{p}-d_{\mathcal{T}}-\|\gamma\| c_{J}\left(1+\|\gamma u\|_{\mathcal{E}}^{\theta-1}\right)\|u\|_{\mathcal{V}} \\
& =m_{\mathcal{T}}\|u\|_{\mathcal{V}}^{p}-\|\gamma\|^{\theta} c_{J}\|u\|_{\mathcal{V}}^{\theta}-d_{\mathcal{T}}-\|\gamma\| c_{J}\|u\|_{\mathcal{V}}
\end{aligned}
$$

If $\theta<p$, from (7) it is obvious that $\mathcal{F}$ is coercive. When $\theta=p$, the assumption $m_{\mathcal{T}}>\|\gamma\|^{p} c_{J}$ in $H(\mathcal{T})$ and $(7)$ render that $\mathcal{F}$ is coercive.

We claim that $\partial_{C} \Phi: \mathcal{V} \rightarrow 2^{\mathcal{V}^{*}}$ is strongly quasi-bounded. As noted in Theorem $3, \partial_{C} \Phi: \mathcal{V} \rightarrow 2^{\mathcal{V}^{*}}$ is a maximal monotone operator. We have $0 \in \operatorname{int} D\left(\partial_{C} \Phi\right)$ owing to hypotheses $H(\varphi)$ and $H(\mathcal{K})$ (ii) in conjunction with int $D(\Phi) \subset \operatorname{int} D\left(\partial_{C} \Phi\right)$. Then, Proposition 6 establishes the validity of the claim.

We are now in a position to apply Theorem 7 ensuring that the inclusion problem (6) has at least a solution. As easily noticed from (1), this guarantees the existence of solutions to Problem 2.

(ii) Given $w \in \mathcal{X}$, let $\left\{u_{n}\right\} \subset \mathcal{S}(w)$ satisfy $u_{n} \rightarrow u$ in $\mathcal{V}$ as $n \rightarrow \infty$. Then, $u \in \mathcal{K}(w)$, thanks to the closedness of $\mathcal{K}(w)$ and there exists $u_{n}^{*} \in \mathcal{T}\left(u_{n}\right)$ such that

$$
\begin{aligned}
& \left\langle\mathcal{L} u_{n}+u_{n}^{*}-f, v-u_{n}\right\rangle \mathcal{V}+J^{0}\left(\gamma u_{n} ; \gamma\left(v-u_{n}\right)\right) \\
& +\varphi(v)-\varphi\left(u_{n}\right) \geq 0 \text { for all } v \in \mathcal{K}(w) .
\end{aligned}
$$

By Lemma 9, the sequence $\left\{\mathcal{L} u_{n}\right\}$ is bounded in $\mathcal{V}^{*}$. Therefore, up to a subsequence, we have $\mathcal{L} u_{n} \rightarrow \mathcal{L} u$ weakly in $\mathcal{V}^{*}$ because the graph of the linear and maximal monotone operator $\mathcal{L}$ is weakly closed. The boundedness of $\mathcal{T}$ allows us to suppose that $u_{n}^{*} \rightarrow u^{*}$ weakly in $\mathcal{V}^{*}$ for some $u^{*} \in \mathcal{V}^{*}$. Inserting $v=u$ in (8) gives

$$
\begin{aligned}
& \limsup _{n \rightarrow \infty}\left\langle u_{n}^{*}, u_{n}-u\right\rangle_{\mathcal{V}} \leq \limsup _{n \rightarrow \infty}\left\langle\mathcal{L} u_{n}-f, u-u_{n}\right\rangle_{\mathcal{V}} \\
& +\limsup _{n \rightarrow \infty} J^{0}\left(\gamma u_{n} ; \gamma\left(u-u_{n}\right)\right)+\varphi(u)-\liminf _{n \rightarrow \infty} \varphi\left(u_{n}\right) \leq 0,
\end{aligned}
$$

where the lower semicontinuity of $\varphi$ and the upper semicontinuity of $J^{0}$ have been used. At this point, the pseudomonotonicity of $\mathcal{T}$ entails $u^{*} \in \mathcal{T}(u)$ and $\left\langle u_{n}^{*}, u_{n}\right\rangle \mathcal{V} \rightarrow$ $\left\langle u^{*}, u\right\rangle_{\mathcal{V}}$. Letting $n \rightarrow \infty$ in (8) leads to $u \in \mathcal{S}(w)$.

(iii) The proof will be carried out by a contrapositive argument. Assume that there are sequences $\left\{u_{n}\right\} \subset D(\mathcal{L})$ and $\left\{w_{n}\right\} \subset \mathcal{X}$ with $u_{n} \in \mathcal{S}\left(w_{n}\right)$ for each $n \in \mathbb{N}$ such that $\left\|u_{n}\right\| \mathcal{V} \rightarrow+\infty$ as $n \rightarrow \infty$. There exists $u_{n}^{*} \in \mathcal{T} u_{n}$ with

$$
\begin{aligned}
& \left\langle\mathcal{L} u_{n}+u_{n}^{*}-f, v-u_{n}\right\rangle \mathcal{V}+J^{0}\left(\gamma u_{n} ; \gamma\left(v-u_{n}\right)\right) \\
& +\varphi(v)-\varphi\left(u_{n}\right) \geq 0 \text { for all } v \in \mathcal{K}\left(w_{n}\right) .
\end{aligned}
$$


Since $0 \in \mathcal{K}(w)$ for all $w \in \mathcal{X}$, we can set $v=0$ to derive

$$
\left\langle\mathcal{L} u_{n},-u_{n}\right\rangle_{\mathcal{V}} \geq\left\langle u_{n}^{*}-f, u_{n}\right\rangle_{\mathcal{V}}+\left\langle\xi_{n}, \gamma u_{n}\right\rangle_{\mathcal{E}}+\varphi\left(u_{n}\right)-\varphi(0)
$$

where $\xi_{n} \in \partial J\left(\gamma u_{n}\right)$ verifies $\left\langle\xi_{n},-\gamma\left(u_{n}\right)\right\rangle_{\mathcal{E}}=J^{0}\left(\gamma u_{n} ; \gamma\left(-u_{n}\right)\right)$. The monotonicity of $\mathcal{L}$, hypotheses $H(\mathcal{T})$ and $H(J)$, and the existence of an affine bound from below for the function $\varphi$ (see, e.g., [2, Proposition 1.10]) yield

$$
\begin{aligned}
0 \geq & \left\langle u_{n}^{*}-f, u_{n}\right\rangle_{\mathcal{V}}+\left\langle\xi_{n}, \gamma u_{n}\right\rangle_{\mathcal{E}}+\varphi\left(u_{n}\right)-\varphi(0) \\
\geq & m_{\mathcal{T}}\left\|u_{n}\right\|_{\mathcal{V}}^{p}-d_{\mathcal{T}}-\|f\|_{\mathcal{V}^{*}}\left\|u_{n}\right\|_{\mathcal{V}}-\|\gamma\| c_{J}\left(1+\left\|\gamma u_{n}\right\|_{\mathcal{E}}^{\theta-1}\right)\left\|u_{n}\right\|_{\mathcal{V}} \\
& -c_{\varphi}\left\|u_{n}\right\|-d_{\varphi}-\varphi(0)
\end{aligned}
$$

with positive constants $c_{\varphi}, d_{\varphi}$, which is a contradiction as $\left\|u_{n}\right\|_{\mathcal{V}} \rightarrow \infty$. Thus, the set $\mathcal{S}(\mathcal{X})$ is bounded in $\mathcal{V}$.

The next lemma gives the Minty's formulation for Problem 2.

Lemma 11 Besides the conditions of Lemma 10, additionally assume that the map $u \in \mathcal{V} \mapsto \mathcal{T} u+\gamma^{*} \partial J(\gamma u) \in 2^{\mathcal{V}^{*}}$ is monotone. Then, the following assertions are valid:

(i) For each $w \in \mathcal{X}, u \in \mathcal{K}(w) \cap D(\mathcal{L})$ is a solution to Problem 2, if and only if,

$$
\left\langle\mathcal{L} v+v^{*}-f, v-u\right\rangle_{\mathcal{V}}+\langle\xi, \gamma(v-u)\rangle_{\mathcal{E}}+\varphi(v)-\varphi(u) \geq 0
$$

for all $v^{*} \in \mathcal{T} v$, all $\xi \in \partial J(\gamma v)$ and all $v \in \mathcal{K}(w) \cap D(\mathcal{L})$.

(ii) For each $w \in \mathcal{X}$, the set $\mathcal{S}(w)$ is convex.

Proof (i) We fix $w \in \mathcal{X}$, and assume that $u \in \mathcal{K}(w) \cap D(\mathcal{L})$ is a solution to Problem 2. Then, with an element $u^{*} \in \mathcal{T}(u)$, we have

$\left\langle\mathcal{L} u+u^{*}-f, v-u\right\rangle_{\mathcal{V}}+J^{0}(\gamma u ; \gamma(v-u))+\varphi(v)-\varphi(u) \geq 0$ for all $v \in \mathcal{K}(w)$.

Notice that $J^{0}(\gamma u ; \gamma(v-u))=\max \left\{\langle\xi, \gamma(v-u)\rangle_{\mathcal{E}}: \xi \in \partial J(\gamma u)\right\}$ whenever $v \in \mathcal{V}$ (see Proposition 4(d)).

Let $z \in \mathcal{K}(w) \cap D(\mathcal{L})$. The monotonicity of $\mathcal{L}$ and $u \mapsto \mathcal{T} u+\gamma^{*} \partial J(\gamma u)$ provide

$$
\left\langle\mathcal{L} z+z^{*}-f, z-u\right\rangle_{\mathcal{V}}+\langle\xi, \gamma(z-u)\rangle_{\mathcal{E}}+\varphi(z)-\varphi(u) \geq 0,
$$

for all $z^{*} \in \mathcal{T} z$ and $\xi \in \partial J(\gamma z)$. We thus arrive at (9).

For the converse, we assume that $u \in \mathcal{K}(w) \cap D(\mathcal{L})$ solves (9). For any $z \in$ $\mathcal{K}(w) \cap D(\mathcal{L})$ and $t \in(0,1)$, we note that $v_{t}=t z+(1-t) u$ is admissible as test function in (9), which for some $v_{t}^{*} \in \mathcal{T} v_{t}$ yields

$$
\left\langle t \mathcal{L} z+(1-t) \mathcal{L} u+v_{t}^{*}-f, z-u\right\rangle \mathcal{V}+J^{0}\left(\gamma v_{t} ; \gamma(z-u)\right)+\varphi(z)-\varphi(u) \geq 0
$$


due to the positive homogeneity of $J^{0}\left(v_{t} ; \cdot\right)$ and the convexity of $\varphi$.

The boundedness of $\mathcal{T}$ and the reflexivity of $\mathcal{V}^{*}$ ensure that along a sequence $v_{t}^{*} \rightarrow z^{*}$ weakly in $\mathcal{V}^{*}$ as $t \rightarrow 0$ for some $z^{*} \in \mathcal{V}^{*}$. The graph of $\mathcal{T}$ is strongly-weakly closed owing to the pseudomonotonicity and boundedness of $\mathcal{T}$, thereby $z^{*} \in \mathcal{T} u$. Due to the compactness of $\gamma$ and upper semicontinuity of $J^{0}$, we can pass to the upper limit as $t \rightarrow 0$ in (10), so that for each $z \in \mathcal{K}(w) \cap D(\mathcal{L})$, there is $z^{*} \in \mathcal{T}(u)$ with

$$
\left\langle\mathcal{L} u+z^{*}-f, z-u\right\rangle_{\mathcal{V}}+J^{0}(\gamma u ; \gamma(z-u))+\varphi(z)-\varphi(u) \geq 0
$$

The density of $D(\mathcal{L})$ in $\mathcal{V}$, in conjunction with the continuity of $\varphi$ on $\mathcal{K}(w)$, hypothesis $H(\varphi)$, the fact that $J^{0}$ is upper semicontinuous, and the assumption that $\mathcal{T}$ is stronglyweakly closed, for each $z \in \mathcal{K}(w)$, there is an element $z^{*} \in \mathcal{T}(u)$ for which we have

$$
\left\langle\mathcal{L} u+z^{*}-f, z-u\right\rangle \mathcal{V}+J^{0}(\gamma u ; \gamma(z-u))+\varphi(z)-\varphi(u) \geq 0 .
$$

We are now prepared to prove that $u \in \mathcal{S}(w)$. For this, assume that $u$ is not a solution to Problem 2. Then, for each $u^{*} \in \mathcal{T}(u)$, there exists $v \in \mathcal{K}(w)$ such that

$$
\left\langle\mathcal{L} u+u^{*}-f, v-u\right\rangle_{\mathcal{V}}+J^{0}(\gamma u ; \gamma(v-u))+\varphi(v)-\varphi(u)<0 .
$$

Denote $\mathcal{R}(u)=\mathcal{T}(u)+\gamma^{*} \partial J(\gamma u)$. Then, by Proposition 4 , for each $v^{*} \in \mathcal{R}(u)$ there exists $v \in \mathcal{K}(w)$ satisfying

$$
\left\langle v^{*}, v-u\right\rangle_{\mathcal{V}}<\varphi(u)-\varphi(v)+\langle f-\mathcal{L} u, v-u\rangle_{\mathcal{V}}
$$

Given $v \in \mathcal{K}(w)$, we introduce the set

$$
\mathcal{Q}_{v}:=\left\{v^{*} \in \mathcal{R}(u) \mid\left\langle v^{*}, v-u\right\rangle_{\mathcal{V}}<\varphi(u)-\varphi(v)+\langle f-\mathcal{L} u, v-u\rangle_{\mathcal{V}}\right\},
$$

which is weakly open in $\mathcal{V}^{*}$. As already observed, $\left\{\mathcal{Q}_{v}\right\}_{v \in \mathcal{K}(w)}$ is an open covering of $\mathcal{R}(u)$. Since $\mathcal{R}(u)$ is weakly compact in $\mathcal{V}^{*}$, a finite sub-covering $\left\{\mathcal{Q}_{v_{1}}, \mathcal{Q}_{v_{2}}, \ldots, \mathcal{Q}_{v_{n}}\right\}$ can be found corresponding to the points $v_{1}, v_{2}, \ldots, v_{n} \in \mathcal{K}(w)$. Let $\kappa_{1}, \kappa_{2}, \ldots, \kappa_{n}$ be a subordinate partition of unity on $\mathcal{R}(u)$ (see, e.g., [12, Lemma 7.3]), that is, for each $i=1,2, \ldots, n, \kappa_{i}: \mathcal{R}(u) \rightarrow[0,1]$ is a weakly continuous function with the support in $\mathcal{Q}_{v_{i}}$ and

$$
\sum_{i=1}^{n} \kappa_{i}\left(v^{*}\right)=1 \text { for all } v^{*} \in \mathcal{R}(u)
$$

We also introduce the map $\mathcal{N}: \mathcal{R}(u) \rightarrow \mathcal{K}(w)$ by

$$
\mathcal{N}\left(v^{*}\right)=\sum_{i=1}^{n} \kappa_{i}\left(v^{*}\right)\left(v_{i}\right) \text { for all } v^{*} \in \mathcal{R}(u)
$$


which is weakly continuous complying with $\kappa_{i}$ for $i=1,2, \ldots, n$. By (12) and the convexity of $\varphi$, for any $v^{*} \in \mathcal{R}(u)$, we have

$$
\begin{aligned}
\left\langle v^{*}, \mathcal{N}\left(v^{*}\right)-u\right\rangle_{\mathcal{V}} & =\sum_{i=1}^{n} \kappa_{i}\left(v^{*}\right)\left\langle v^{*}, v_{i}-u\right\rangle_{\mathcal{V}} \\
& <\varphi(u)-\varphi\left(\sum_{i=1}^{n} \kappa_{i}\left(v^{*}\right) v_{i}\right)+\left\langle f-\mathcal{L} u, \sum_{i=1}^{n} \kappa_{i}\left(v^{*}\right) v_{i}-u\right\rangle_{\mathcal{V}} \\
& =\varphi(u)-\varphi\left(\mathcal{N}\left(v^{*}\right)\right)+\left\langle f-\mathcal{L} u, \mathcal{N}\left(v^{*}\right)-u\right\rangle_{\mathcal{V}}
\end{aligned}
$$

Let us define the maps $\Upsilon: \mathcal{K}(w) \rightarrow 2^{\mathcal{R}(u)}$ by

$$
\Upsilon(v):=\left\{v^{*} \in \mathcal{R}(u) \mid\left\langle v^{*}, v-u\right\rangle_{\mathcal{V}} \geq \varphi(u)-\varphi(v)+\langle f-\mathcal{L} u, v-u\rangle_{\mathcal{V}}\right\}
$$

for all $v \in \mathcal{K}(w)$ and $\Psi: \mathcal{R}(u) \rightarrow 2^{\mathcal{R}(u)}$ by

$$
\Psi\left(v^{*}\right):=\Upsilon\left(\mathcal{N}\left(v^{*}\right)\right) \text { for all } v^{*} \in \mathcal{R}(u) .
$$

By means of (11), it is seen that $\Upsilon$ has nonempty, weakly compact, and convex values. We prove that $\Upsilon$ is upper semicontinuous from the norm topology of $\mathcal{V}$ to the weak topology of $\mathcal{V}^{*}$. Making use of a classical result (see, e.g., [37, Proposition 3.8]), this amounts to check that for each weakly closed set $B$ in $\mathcal{V}^{*}$, the set

$$
\Upsilon^{-}(B):=\{v \in \mathcal{K}(w) \mid \Upsilon(v) \cap B \neq \emptyset\},
$$

is closed in $\mathcal{V}$. Let $\left\{v_{n}\right\} \subset \Upsilon^{-}(B)$ satisfy $v_{n} \rightarrow v$ in $\mathcal{V}$ as $n \rightarrow \infty$, for some $v \in \mathcal{V}$. For each $n \in \mathbb{N}$, we can find $v_{n}^{*} \in \mathcal{R}(u) \cap B$ such that

$$
\left\langle v_{n}^{*}, v_{n}-u\right\rangle_{\mathcal{V}} \geq \varphi(u)-\varphi\left(v_{n}\right)+\left\langle f-\mathcal{L} u, v_{n}-u\right\rangle_{\mathcal{V}}
$$

Through the weak compactness of $\mathcal{R}(u)$, we may suppose that $v_{n}^{*} \rightarrow v^{*} \in B$ weakly in $\mathcal{V}^{*}$ as $n \rightarrow \infty$, for some $v^{*} \in \mathcal{R}(u)$. Now pass to the upper limit as $n \rightarrow \infty$ in (14) and use the lower semicontinuity of $\varphi$ to get

$$
\left\langle v^{*}, v-u\right\rangle_{\mathcal{V}} \geq \varphi(u)-\varphi(v)+\langle f-\mathcal{L} u, v-u\rangle_{\mathcal{V}}
$$

which means that $v^{*} \in \Upsilon(v) \cap B$, thus proving the desired property for $\Upsilon$. As a consequence, through the weak continuity of $\mathcal{N}$ we infer that $\Psi$ is also stronglyweakly upper semicontinuous (refer, e.g., to [19, Theorem 1.2.8]).

The preceding arguments enable us to apply Tychonov's fixed point principle (see, e.g., [12, Theorem 8.6]) to the map $\Psi$. Hence, there exists $v^{*} \in \mathcal{R}(u)$ such that

$$
\left\langle v^{*}, \mathcal{N}\left(v^{*}\right)-u\right\rangle_{\mathcal{V}} \geq \varphi(u)-\varphi\left(\mathcal{N}\left(v^{*}\right)\right)+\left\langle f-\mathcal{L} u, \mathcal{N}\left(v^{*}\right)-u\right\rangle_{\mathcal{V}}
$$

This contradicts (13), thus establishing that $u \in \mathcal{K}(w)$ solves Problem 2. 
(ii) Let $u_{1}, u_{2} \in \mathcal{S}(w)$ and $t \in(0,1)$. On the basis of part (i), for $i=1,2$ we have

$$
\left\langle\mathcal{L} v+v^{*}-f, v-u_{i}\right\rangle \mathcal{V}+\left\langle\xi, \gamma\left(v-u_{i}\right)\right\rangle_{\mathcal{E}}+\varphi(v)-\varphi\left(u_{i}\right) \geq 0
$$

for all $v^{*} \in \mathcal{T} v, \xi \in \partial J(\gamma v)$ and $v \in \mathcal{K}(w) \cap D(\mathcal{L})$. Set $u_{t}=t u_{1}+(1-t) u_{2}$. Then, the convexity of $\varphi$ implies

$$
\left\langle\mathcal{L} v+v^{*}-f+\gamma^{*} \xi, v-u_{t}\right\rangle \mathcal{V}+\varphi(v)-\varphi\left(u_{t}\right) \geq 0
$$

for all $v^{*} \in \mathcal{T} v, \xi \in \partial J(\gamma v)$ and $v \in \mathcal{K}(w) \cap D(\mathcal{L})$. Invoking part (i), we infer that $u_{t}$ is a solution to Problem 2, so the set $\mathcal{S}(w)$ is convex.

The above preparation permits us to give the following existence result for Problem 1.

Theorem 12 Assume that $H(0), H(\mathcal{L}), H(\mathcal{T}), H(\gamma), H(J), H(\varphi)$, and $H(\mathcal{K})$ are fulfilled. If, in addition, $u \in \mathcal{V} \mapsto \mathcal{T} u+\gamma^{*} \partial J(\gamma u) \in 2^{\mathcal{V}^{*}}$ is monotone, then the set of solutions to Problem 1 is nonempty and sequentially weakly compact in $\mathcal{V}$.

Proof To prove that the set of solutions to Problem 1 is nonempty, we apply Theorem 8 to the set-valued mapping $\mathcal{S}: \mathcal{X} \rightarrow 2^{\mathcal{X}}$. We first check that the graph $\operatorname{Gr}(\mathcal{S})$ of $\mathcal{S}$ is sequentially weakly closed in $\mathcal{V} \times \mathcal{V}$. Let $\left\{\left(w_{n}, u_{n}\right)\right\} \subset G r(\mathcal{S})$ satisfy $\left(w_{n}, u_{n}\right) \rightarrow$ $(w, u)$ weakly in $\mathcal{V} \times \mathcal{V}$ for some $(w, u) \in \mathcal{V} \times \mathcal{V}$. The fact that $u_{n} \in \mathcal{S}\left(w_{n}\right)$ means that there is $u_{n}^{*} \in \mathcal{T} u_{n}$ such that

$$
\left\langle\mathcal{L} u_{n}+u_{n}^{*}-f, v-u_{n}\right\rangle_{\mathcal{V}}+J^{0}\left(\gamma u_{n} ; \gamma\left(v-u_{n}\right)\right)+\varphi(v)-\varphi\left(u_{n}\right) \geq 0 \text { for all } v \in \mathcal{K}\left(w_{n}\right) .
$$

The boundedness of $\mathcal{T}$ allows us to suppose that up to a subsequence $u_{n}^{*} \rightarrow u^{*}$ weakly in $\mathcal{V}^{*}$ as $n \rightarrow \infty$, for some $u^{*} \in \mathcal{V}^{*}$.

It follows from Lemma 9 that the sequence $\left\{\mathcal{L} u_{n}\right\}$ is bounded in $\mathcal{V}^{*}$. Note that $\mathcal{L}$ is linear and maximal monotone, so it is weakly closed graph. This combined with the convergence $u_{n} \rightarrow u$ weakly in $\mathcal{V}$ entails $u \in D(\mathcal{L})$ and $\mathcal{L}\left(u_{n}\right) \rightarrow \mathcal{L}(u)$ weakly in $\mathcal{V}^{*}$. Then, hypothesis $H(\mathcal{K})$ (iii) implies $u \in \mathcal{K}(w)$. In turn, condition $H(\mathcal{K})$ (iv) provides a sequence $\left\{z_{n}\right\} \subset \mathcal{V}$ with $z_{n} \in \mathcal{K}\left(w_{n}\right)$ and $z_{n} \rightarrow u$ as $n \rightarrow \infty$.

Upon inserting $v=z_{n}$ in (15), we find

$$
\begin{aligned}
& \left\langle\mathcal{L} u_{n}-f, z_{n}-u_{n}\right\rangle_{\mathcal{V}}+\left\langle u_{n}^{*}, z_{n}-u\right\rangle_{\mathcal{V}}+J^{0}\left(\gamma u_{n} ; \gamma\left(z_{n}-u_{n}\right)\right) \\
& +\varphi\left(z_{n}\right)-\varphi\left(u_{n}\right) \geq\left\langle u_{n}^{*}, u_{n}-u\right\rangle_{\mathcal{V}}
\end{aligned}
$$

The monotonicity of $\mathcal{L}$ ensures

$$
0 \leq \liminf _{n \rightarrow \infty}\left\langle\mathcal{L} u_{n}-\mathcal{L} u, u_{n}-u\right\rangle_{\mathcal{V}}=\liminf _{n \rightarrow \infty}\left\langle\mathcal{L} u_{n}, u_{n}\right\rangle_{\mathcal{V}}-\langle\mathcal{L} u, u\rangle_{\mathcal{V}}
$$

which gives

$$
\limsup _{n \rightarrow \infty}\left\langle\mathcal{L} u_{n}, z_{n}-u_{n}\right\rangle \mathcal{V} \leq \limsup _{n \rightarrow \infty}\left\langle\mathcal{L} u_{n}, z_{n}\right\rangle \mathcal{V}-\liminf _{n \rightarrow \infty}\left\langle\mathcal{L} u_{n}, u_{n}\right\rangle \mathcal{V} \leq 0
$$


The compactness of $\gamma$ and the upper semicontinuity of $(u, v) \mapsto J^{0}(u ; v)$ yield

$$
\limsup _{n \rightarrow \infty} J^{0}\left(\gamma u_{n} ; \gamma\left(z_{n}-u_{n}\right)\right) \leq 0
$$

By $H(\varphi)$, we have $\bigcup_{w \in \mathcal{X}} K(w) \subset \operatorname{int} D(\varphi)$, so $\varphi$ is continuous on $\bigcup_{w \in \mathcal{X}} K(w)$, resulting in

$$
\limsup _{n \rightarrow \infty}\left[\varphi\left(z_{n}\right)-\varphi\left(u_{n}\right)\right] \leq \limsup _{n \rightarrow \infty} \varphi\left(z_{n}\right)-\liminf _{n \rightarrow \infty} \varphi\left(u_{n}\right) \leq 0 .
$$

Letting $n \rightarrow \infty$ in (16) and using (17)-(19), we get

$$
\limsup _{n \rightarrow \infty}\left\langle u_{n}^{*}, u_{n}-u\right\rangle_{\mathcal{V}} \leq 0
$$

The latter combined with the pseudomonotonicity of $\mathcal{T}$ results in

$$
u^{*} \in \mathcal{T} u \quad \text { and } \quad\left\langle u_{n}^{*}, u_{n}\right\rangle \mathcal{V} \rightarrow\left\langle u^{*}, u\right\rangle_{\mathcal{V}}
$$

Let $v \in \mathcal{K}(w)$. By hypothesis $H(\mathcal{K})\left(\right.$ iv), up to a subsequence of $\left\{w_{n}\right\}$, there is a sequence $\left\{v_{n}\right\} \subset \mathcal{V}$ with $v_{n} \in \mathcal{K}\left(w_{n}\right)$ and $v_{n} \rightarrow v$ as $n \rightarrow \infty$. Taking $v=v_{n}$ in (15), it turns out from (17)-(20) that $(w, u) \in G r(\mathcal{S})$ since

$$
\begin{aligned}
0 \leq & \limsup _{n \rightarrow \infty}\left[\left\langle\mathcal{L} u_{n}+u_{n}^{*}-f, v_{n}-u_{n}\right\rangle \mathcal{V}+J^{0}\left(\gamma u_{n} ; \gamma\left(v_{n}-u_{n}\right)\right)+\varphi\left(v_{n}\right)-\varphi\left(u_{n}\right)\right] \\
\leq & \limsup _{n \rightarrow \infty}\left\langle\mathcal{L} u_{n}+u_{n}^{*}-f, v_{n}-u_{n}\right\rangle_{\mathcal{V}}+\limsup _{n \rightarrow \infty} J^{0}\left(\gamma u_{n} ; \gamma\left(v_{n}-u_{n}\right)\right) \\
& +\limsup _{n \rightarrow \infty} \varphi\left(v_{n}\right)-\liminf _{n \rightarrow \infty} \varphi\left(u_{n}\right) \\
& \leq\left\langle\mathcal{L} u+u^{*}-f, v-u\right\rangle_{\mathcal{V}}+J^{0}(\gamma u ; \gamma(v-u))+\varphi(v)-\varphi(u) .
\end{aligned}
$$

Therefore, the graph $\operatorname{Gr}(\mathcal{S})$ of $\mathcal{S}$ is sequentially weakly closed in $\mathcal{V} \times \mathcal{V}$.

The other requirements needed to apply Theorem 8 for $\mathcal{S}$ are fulfilled according to Lemmas 10 and 11 (ii). We infer from Theorem 8 that $\mathcal{S}$ has at least a fixed point in $\mathcal{X}$; thus, there exists a solution to Problem 1 .

Now, we proceed to show that the set of solutions to Problem 1 is sequentially weakly compact. Let $\left\{u_{n}\right\}$ be a sequence of solutions to Problem 1 . Then, $u_{n} \in \mathcal{K}\left(u_{n}\right)$, and for some $u_{n}^{*} \in \mathcal{T}\left(u_{n}\right)$, we have

$$
\left\langle\mathcal{L} u_{n}+u_{n}^{*}-f, v-u_{n}\right\rangle_{\mathcal{V}}+J^{0}\left(\gamma u_{n} ; \gamma\left(v-u_{n}\right)\right)+\varphi(v)-\varphi\left(u_{n}\right) \geq 0
$$

for all $v \in \mathcal{K}\left(u_{n}\right)$. The reasoning in the proof of Lemma 10(iii) reveals that the sequence $\left\{u_{n}\right\}$ is bounded. Without loss of generality, we may assume that $u_{n} \rightarrow u$ weakly in $\mathcal{V}$ for some $u \in \mathcal{V}$. Since $u_{n} \in \mathcal{S}\left(u_{n}\right)$, by Lemma 9 the sequence $\left\{\mathcal{L} u_{n}\right\}$ is bounded in $\mathcal{V}^{*}$. The reflexivity of $\mathcal{V}^{*}$ and because $\mathcal{L}$ is linear and closed, we find along a subsequence that $\mathcal{L} u_{n} \rightarrow \mathcal{L} u$ weakly in $\mathcal{V}^{*}$ as $n \rightarrow \infty$. Then, using hypothesis $H(\mathcal{K})($ iii), it is true that $u \in \mathcal{K}(u)$. 
By virtue of condition $H(\mathcal{K})$ (iv), possibly for a subsequence of $\left\{u_{n}\right\}$, there is $\left\{z_{n}\right\} \subset$ $\mathcal{X}$ such that $z_{n} \in \mathcal{K}\left(u_{n}\right)$ and $z_{n} \rightarrow u$. As $\mathcal{T}$ is a bounded mapping, the sequence $\left\{u_{n}^{*}\right\}$ is bounded in $\mathcal{V}^{*}$, so we can suppose that $u_{n}^{*} \rightarrow u^{*}$ weakly in $\mathcal{V}$ for some $u^{*} \in \mathcal{V}^{*}$.

Insert $v=z_{n}$ in (21) and use (17)-(19) to deduce that

$$
\limsup _{n \rightarrow \infty}\left\langle u_{n}^{*}, u_{n}-u\right\rangle_{\mathcal{V}} \leq 0
$$

Due to the pseudomonotonicity of $\mathcal{T}$, we derive $u^{*} \in \mathcal{T} u$ and $\lim _{n \rightarrow \infty}\left\langle u_{n}^{*}, u_{n}\right\rangle \mathcal{V}=$ $\left\langle u^{*}, u\right\rangle \mathcal{V}$.

For any $v \in \mathcal{K}(u)$, by assumption $H(\mathcal{K})\left(\right.$ iv) we are able to find a sequence $\left\{v_{n}\right\}$ with $v_{n} \in \mathcal{K}\left(u_{n}\right)$ and $v_{n} \rightarrow v$ in $\mathcal{V}$. Passing to the limit in (21) with $v_{n}$ as test element yields

$$
\left\langle\mathcal{L} u+u^{*}-f, v-u\right\rangle_{\mathcal{V}}+J^{0}(\gamma u ; \gamma(v-u))+\varphi(v)-\varphi(u) \geq 0,
$$

so $u$ is a solution to Problem 1. Consequently, the set of solutions to Problem 1 is sequentially weakly compact.

\section{An Optimal Control Problem}

In this section, we focus on an optimal control problem associated with the (QVHVI). In the following, we continue to adhere to the notation used in Problem 1. Additionally, let $\mathcal{W}$ be a reflexive Banach space which is compactly embedded in $\mathcal{V}^{*}$ and let $\mathcal{B}$ be a nonempty and weakly closed subset of $\mathcal{W}$. Consider the set-valued mapping $\Pi: \mathcal{V}^{*} \rightarrow 2^{\mathcal{X}}$ defined by

$$
\Pi(f):=\left\{u \in D(\mathcal{L}) \mid u \text { is a solution to Problem } 1 \text { corresponding to } f \in \mathcal{V}^{*}\right\} .
$$

We also need to introduce functions $g: \mathcal{X} \rightarrow \mathbb{R}$ and $h: \mathcal{B} \rightarrow \mathbb{R}$ that satisfy the following:

$H(g): g: \mathcal{X} \rightarrow \mathbb{R}$ is bounded from below and sequentially weakly lower semicontinuous.

$H(h): h: \mathcal{B} \rightarrow \mathbb{R}$ is bounded from below, sequentially weakly lower semicontinuous and coercive, that is, $h(f) \rightarrow+\infty$ as $\|f\|_{\mathcal{W}} \rightarrow+\infty$.

Our focus is on the following nonlinear optimal control problem:

Problem 3 Find $f \in \mathcal{B}$ such that

$$
F(f)=\inf _{l \in \mathcal{B}} F(l) \text { with } F(l):=\inf _{u \in \Pi(l)} g(u)+h(l),
$$

where $\Pi(l)$ is the set of solutions to Problem 1 corresponding to $l \in \mathcal{V}^{*}$.

To give an existence result for the above control problem, we begin with the following:

Lemma 13 Under the assumptions of Theorem 12, the following statements hold: 
(i) $\Pi: \mathcal{V}^{*} \rightarrow 2^{\mathcal{X}}$ is a bounded map.

(ii) If $\left\{f_{n}\right\} \subset \mathcal{V}^{*}$ is such that $f_{n} \rightarrow f$ in $\mathcal{V}^{*}$ as $n \rightarrow \infty$ for some $f \in \mathcal{V}^{*}$, then

$$
w-\limsup _{n \rightarrow \infty} \Pi\left(f_{n}\right) \subset \Pi(f),
$$

where $w$ - lim $\sup _{n \rightarrow \infty} \Pi\left(f_{n}\right)$ stands for the sequential Kuratowski upper limit of $\left\{\Pi\left(f_{n}\right)\right\}$ with respect to the weak topology of $\mathcal{V}$, namely,

$$
\begin{aligned}
& w-\limsup _{n \rightarrow \infty} \Pi\left(f_{n}\right) \\
& :=\left\{u \in \mathcal{X} \mid \exists u_{n_{k}} \in \Pi\left(f_{n_{k}}\right), u_{n_{k}} \rightarrow u \text { weakly in } \mathcal{V} \text { as } k \rightarrow \infty\right\} .
\end{aligned}
$$

Proof (i) To prove the claim, we begin by assuming that there exists a bounded $\operatorname{set} \mathcal{C}$ in $\mathcal{V}^{*}$ such that $\Pi(\mathcal{C})$ is unbounded. This allows us to find sequences $\left\{f_{n}\right\} \subset \mathcal{C}$ and $\left\{u_{n}\right\} \subset \mathcal{V}$ with $u_{n} \in \Pi\left(f_{n}\right)$ for all $n \in \mathbb{N}$ satisfying $\left\|u_{n}\right\| \mathcal{V} \rightarrow \infty$ as $n \rightarrow \infty$. We have $u_{n} \in \mathcal{K}\left(u_{n}\right)$ and $u_{n}^{*} \in \mathcal{T}\left(u_{n}\right)$ such that

$$
\begin{aligned}
& \left\langle\mathcal{L} u_{n}+u_{n}^{*}-f_{n}, v-u_{n}\right\rangle \mathcal{V}+\varphi(v)-\varphi\left(u_{n}\right) \\
& +J^{0}\left(\gamma u_{n} ; \gamma\left(v-u_{n}\right)\right) \geq 0 \text { for all } v \in \mathcal{K}\left(u_{n}\right) .
\end{aligned}
$$

By assumption $H(\mathcal{K})($ ii), we have $0 \in \mathcal{K}(w)$ for all $w \in \mathcal{X}$, and hence, we can insert $v=0$ in the above inequality. By means of $H(\mathcal{L}), H(\mathcal{T}), H(\gamma), H(J), H(\varphi)$, and the boundedness of $\mathcal{C}$, we get

$$
\begin{aligned}
0= & \left\langle\mathcal{L} 0,-u_{n}\right\rangle \mathcal{V} \geq\left\langle\mathcal{L} u_{n},-u_{n}\right\rangle \mathcal{V} \\
\geq & \left\langle u_{n}^{*}-f_{n}, u_{n}\right\rangle_{\mathcal{V}}-\varphi(0)+\varphi\left(u_{n}\right)-J^{0}\left(\gamma u_{n} ;-\gamma u_{n}\right) \\
\geq & m_{\mathcal{T}}\left\|u_{n}\right\|_{\mathcal{V}}^{p}-d_{\mathcal{T}}-M_{\mathcal{C}}\left\|u_{n}\right\| \mathcal{V}-\|\gamma\| c_{J}\left(1+\left\|\gamma u_{n}\right\|_{\mathcal{E}}^{\theta-1}\right)\left\|u_{n}\right\| \mathcal{V} \\
& -c_{\varphi}\left\|u_{n}\right\|-d_{\varphi}-\varphi(0)
\end{aligned}
$$

with a constant $M_{\mathcal{C}}>0$. Using condition $H(J)$, in the limit as $n \rightarrow \infty$, leads to a contradiction. We conclude that $\Pi: \mathcal{V}^{*} \rightarrow 2^{\mathcal{X}}$ is a bounded map.

(ii) Let $u \in w-\lim \sup _{n \rightarrow \infty} \Pi\left(f_{n}\right)$. Let $\left\{u_{n_{k}}\right\} \subset \mathcal{V}$ with $u_{n_{k}} \in \Pi\left(f_{n_{k}}\right)$ be such that

$$
u_{n_{k}} \rightarrow u \text { weakly in } \mathcal{V} \text { as } k \rightarrow \infty
$$

Then, $u_{n_{k}} \in \mathcal{K}\left(u_{n_{k}}\right)$, and for some $u_{n_{k}}^{*} \in \mathcal{T}\left(u_{n_{k}}\right)$, we have

$$
\begin{aligned}
& \left\langle\mathcal{L} u_{n_{k}}+u_{n_{k}}^{*}, v-u_{n_{k}}\right\rangle \mathcal{V}+\varphi(v)-\varphi\left(u_{n_{k}}\right)+J^{0}\left(\gamma u_{n_{k}} ; \gamma\left(v-u_{n_{k}}\right)\right) \\
& \quad \geq\left\langle f_{n_{k}}, v-u_{n_{k}}\right\rangle \mathcal{V} \text { for all } v \in \mathcal{K}\left(u_{n_{k}}\right) .
\end{aligned}
$$

The boundedness of $\mathcal{T}$ allows us to assume that $u_{n_{k}}^{*} \rightarrow u^{*}$ weakly in $\mathcal{V}^{*}$ for some $u^{*} \in$ $\mathcal{V}^{*}$. The convergence in (23) and condition $H(\mathcal{K})($ iii) imply that $u \in \mathcal{K}(u)$. Lemma 9 
and the fact that $\mathcal{L}$ is linear and graph closed imply $u \in D(\mathcal{L})$ and $\mathcal{L} u_{n_{k}} \rightarrow \mathcal{L} u$ weakly in $\mathcal{V}^{*}$ as $k \rightarrow \infty$. Besides, assumption $H(\mathcal{K})\left(\right.$ iv) provides a sequence $\left\{z_{k}\right\} \subset \mathcal{V}$ with $z_{k} \in \mathcal{K}\left(u_{n_{k}}\right)$ such that $z_{k} \rightarrow u$ in $\mathcal{V}$. Inserting $v=z_{k}$ in (24) yields

$$
\limsup _{n \rightarrow \infty}\left\langle u_{n_{k}}^{*}, u_{n_{k}}-u\right\rangle_{\mathcal{V}} \leq 0
$$

and the pseudomonotonicity of $\mathcal{T}$ further ensures that

$$
u^{*} \in \mathcal{T} u \text { and }\left\langle u_{n_{k}}^{*}, u_{n_{k}}\right\rangle \mathcal{V} \rightarrow\left\langle u^{*}, u\right\rangle_{\mathcal{V}}
$$

Let $z \in \mathcal{K}(u)$. Hypothesis $H(\mathcal{K})$ (iv) guarantees the existence, up to a subsequence, of $v_{k} \in \mathcal{K}\left(u_{n_{k}}\right)$ with $v_{k} \rightarrow z$ as $k \rightarrow \infty$. Set $v=v_{k}$ in (24). Passing to the limit on the pattern of Step 1 in the proof of Theorem 12 leads to

$$
\left\langle\mathcal{L} u+u^{*}, z-u\right\rangle_{\mathcal{V}}+\varphi(z)-\varphi(u)+J^{0}(\gamma u ; \gamma(z-u)) \geq\langle f, z-u\rangle_{\mathcal{V}}
$$

Hence, $u$ is a solution to Problem 1 associated with $f$, that is, $u \in \Pi(f)$.

The following is the existence result for Problem 3:

Theorem 14 Besides the assumptions of Theorem 12, additionally assume the conditions $H(g)$ and $H(h)$. Then, the set of solutions to Problem 3 is nonempty and sequentially weakly compact.

Proof We claim that for each $f \in \mathcal{V}^{*}$, there exists $u \in \Pi(f)$ such that $F(f)=$ $g(u)+h(f)$. To this end, we recall from Theorem 12 that the set $\Pi(f)$ is nonempty and sequentially weakly compact in $\mathcal{V}$. Then, by the Weierstrass minimization theorem, assumption $H(g)$ entitles the existence of $u \in \Pi(f)$ satisfying

$$
g(u)=\inf _{v \in \Pi(f)} g(v),
$$

which proves the claim. Consequently, the function $F: \mathcal{V}^{*} \rightarrow \mathbb{R}$ given in (22) is well defined.

Denote $\theta:=\inf _{f \in \mathcal{B}} F(f)$. Let $\left\{f_{n}\right\} \subset \mathcal{B}$ be a minimizing sequence for $F$, that is,

$$
\lim _{n \rightarrow \infty} F\left(f_{n}\right)=\theta \in[-\infty,+\infty)
$$

Let $u_{n} \in \Pi\left(f_{n}\right)$ be a sequence such that

$$
F\left(f_{n}\right)=g\left(u_{n}\right)+h\left(f_{n}\right) \text { for each } n \in \mathbb{N},
$$

which exists by the arguments used above. Note that the sequence $\left\{f_{n}\right\}$ is bounded in $\mathcal{W}$. If not, we can assume that, along a subsequence, we have

$$
\left\|f_{n}\right\|_{\mathcal{W}} \rightarrow+\infty, \text { as } n \rightarrow \infty
$$


However, by (26) and hypothesis $H(g)$, we have $F\left(f_{n}\right) \geq M_{g}+h\left(f_{n}\right)$, with $M_{g} \in \mathbb{R}$. Applying (27) and the coercivity of $h$ in $H(h)$, we obtain a contradiction to (25):

$$
\lim _{n \rightarrow \infty} F\left(f_{n}\right) \geq M_{g}+\lim _{n \rightarrow \infty} h\left(f_{n}\right)=+\infty
$$

Therefore, the sequence $\left\{f_{n}\right\}$ must be bounded in $\mathcal{W}$.

Due to the reflexivity of $\mathcal{W}$, there exists $f^{*} \in \mathcal{W}$ such that, along a subsequence,

$$
f_{n} \rightarrow f^{*} \text { weakly in } \mathcal{H} \text { as } n \rightarrow \infty \text {, }
$$

with $f^{*} \in \mathcal{B}$, by the sequential weak closedness of $\mathcal{B}$. Since $\left\{u_{n}\right\} \subset \Pi\left(\left\{f_{n}\right\}\right)$, it follows from Lemma 13(i) that $\left\{u_{n}\right\}$ is bounded in $\mathcal{V}$. Without any loss of generality, we may assume

$$
u_{n} \rightarrow \tilde{u} \text { weakly in } \mathcal{V} \text { for some } \tilde{u} \in \mathcal{X}
$$

Note that $\mathcal{W}$ is compactly embedded in $\mathcal{V}^{*}$; thus, by (28), $f_{n} \rightarrow f^{*}$ in $\mathcal{V}^{*}$. Then, from (29) and Lemma 13(ii), we infer that $\tilde{u} \in \Pi\left(f^{*}\right)$. Relying on the sequential weak lower semicontinuity of $h$ and $g$, in conjunction with (25) and (26), we obtain

$$
\begin{aligned}
\theta=\inf _{f \in \mathcal{B}} F(f) \leq F\left(f^{*}\right) & =\inf _{u \in \Pi\left(f^{*}\right)} g(u)+h\left(f^{*}\right) \\
& \leq g(\widetilde{u})+h\left(f^{*}\right) \\
& \leq \liminf _{n \rightarrow \infty} g\left(u_{n}\right)+\liminf _{n \rightarrow \infty} h\left(f_{n}\right) \\
& \leq \liminf _{n \rightarrow \infty}\left[g\left(u_{n}\right)+h\left(f_{n}\right)\right] \\
& =\liminf _{n \rightarrow \infty} F\left(f_{n}\right)=\theta .
\end{aligned}
$$

This establishes that $f \in \mathcal{B}$ is a solution to Problem 3 .

It remains to show that the set of solutions to Problem 3 is sequentially weakly compact. Let $\left\{f_{n}\right\}$ be a sequence of solutions to Problem 3. Hypotheses $H(g)$ and $H(h)$ determine the coercivity of the cost function $F$; thus, the sequence $\left\{f_{n}\right\}$ is bounded in $\mathcal{W}$. Therefore, we can find $f^{*} \in \mathcal{W}$ such that, for a subsequence, convergence (28) holds. There is a sequence $u_{n} \in \Pi\left(f_{n}\right)$ that verifies (26). From Lemma 13(i), we may assume that (29) is valid with some $\tilde{u} \in \Pi\left(f^{*}\right)$. Carrying out the same reasoning as in (30), we conclude that $f^{*}$ is a solution to Problem 3 . The proof is thus complete.

\section{Concluding Remarks}

We studied a new class of evolutionary quasi-variational-hemivariational inequalities. The main contribution is a new existence result for the considered inequality problem and the solvability of an associated optimal control problem. It is of genuine interest to study the impact of data contamination on the optimal control problem by developing a regularization framework for the stable approximation. We plan to address this in future work. 
Acknowledgements The authors are immensely grateful to the reviewers for their meticulous reading and helpful suggestions that brought substantial improvements to the manuscript. This project has received funding from the NNSF of China Grant Nos. 12001478, 12026255, and 12026256, the European Union's Horizon 2020 Research and Innovation Programme under the Marie Skłodowska-Curie grant agreement No. 823731 CONMECH, National Science Center of Poland under Preludium Project No. 2017/25/N/ST1/00611, and the Startup Project of Doctor Scientific Research of Yulin Normal University No. G2020ZK07. It is also supported by Natural Science Foundation of Guangxi Grants Nos. 2021GXNSFFA196004 and 2020GXNSFBA297137, and the Ministry of Science and Higher Education of Republic of Poland under Grants Nos. 4004/GGPJII/H2020/2018/0 and 440328/PnH2/2019. The third author is supported by the NSF (DMS 1720067).

Availability of data and material Not applicable.

Open Access This article is licensed under a Creative Commons Attribution 4.0 International License, which permits use, sharing, adaptation, distribution and reproduction in any medium or format, as long as you give appropriate credit to the original author(s) and the source, provide a link to the Creative Commons licence, and indicate if changes were made. The images or other third party material in this article are included in the article's Creative Commons licence, unless indicated otherwise in a credit line to the material. If material is not included in the article's Creative Commons licence and your intended use is not permitted by statutory regulation or exceeds the permitted use, you will need to obtain permission directly from the copyright holder. To view a copy of this licence, visit http://creativecommons.org/licenses/by/4.0/.

\section{References}

1. Barboteu, M., Bartosz, K., Han, W., Janiczko, T.: Numerical analysis of a hyperbolic hemivariational inequality arising in dynamic contact. SIAM J Numer. Anal. 53, 527-550 (2015)

2. Brezis, H.: Functional Analysis. Universitext, Springer, New York, Sobolev Spaces and Partial Differential Equations (2011)

3. Browder, F.E., Hess, P.: Nonlinear mappings of monotone type in Banach spaces. J. Funct. Anal. 11, 251-294 (1972)

4. Carl, S., Le, V. K., Motreanu, D.: Nonsmooth variational problems and their inequalities. Comparison principles and applications, Springer Monographs in Mathematics, Springer, New York, (2007)

5. Clarke, F.H.: Optimization and Nonsmooth Analysis. Wiley, New York (1983)

6. Denkowski, Z., Migórski, S., Papageorgiou, N.S.: An Introduction to Nonlinear Analysis: Applications. Kluwer Academic/Plenum Publishers, Boston (2003)

7. Denkowski, Z., Gasiński, L., Papageorgiou, N.S.: Nontrivial solutions for resonant hemivariational inequalities. J. Global Optim. 34, 317-337 (2006)

8. Facchinei, F., Pang, J.S., Scutari, G., Lampariello, L.: VI-constrained hemivariational inequalities: distributed algorithms and power control in ad-hoc networks. Math. Program. 145, 59-96 (2014)

9. Giannessi, F., Khan, A.A.: Regularization of non-coercive quasi-variational inequalities. Control Cybern. 29, 91-110 (2000)

10. Gasiński, L., Migórski, S., Ochal, A.: Existence results for evolution inclusions and variationalhemivariational inequalities. Appl. Anal. 94, 1670-1694 (2015)

11. Gasiński, L.: Existence and multiplicity results for quasilinear hemivariational inequalities at resonance. Math. Nachr. 281, 1728-1746 (2008)

12. Granas, A., Dugundji, J.: Fixed Point Theory. Springer-Verlag, New York (2003)

13. Gwinner, J., Jadamba, B., Khan, A.A., Sama, M.: Identification in variational and quasi-variational inequalities. J. Convex Anal. 25, 545-569 (2018)

14. Gwinner, J.: An optimization approach to parameter identification in variational inequalities of second kind. Optim. Lett. 12, 1141-1154 (2018)

15. Han, W., Sofonea, M., Barboteu, M.: Numerical analysis of elliptic hemivariational inequalities. SIAM J. Numer. Anal. 55, 640-663 (2017)

16. Han, W., Migórski, S., Sofonea, M.: A class of variational-hemivariational inequalities with applications to frictional contact problems. SIAM J. Math. Anal. 46, 3891-3912 (2014) 
17. Hintermüller, M., Kovtunenko, V.A., Kunisch, K.: Obstacle problems with cohesion: a hemivariational inequality approach and its efficient numerical solution. SIAM J. Optim. 21, 491-516 (2011)

18. Huong, N.T.T., Yao, J.C., Yen, N.D.: Polynomial vector variational inequalities under polynomial constraints and applications. SIAM J. Optim. 26, 1060-1071 (2016)

19. Kamenskii, M., Obukhovskii, V., Zecca, P.: Condensing Multivalued Maps and Semilinear Differential Inclusions in Banach Space. Walter de Gruyter, Berlin (2001)

20. Khan, A.A., Motreanu, D.: Existence theorems for elliptic and evolutionary variational and quasivariational inequalities. J. Optim. Theory Appl. 167, 1136-1161 (2015)

21. Khan, A.A., Motreanu, D.: Inverse problems for quasi-variational inequalities. J. Global Optim. 70, 401-411 (2018)

22. Khan, A.A., Sama, M.: Optimal control of multi-valued quasi-variational inequalities. Nonlinear Anal. TMA 75, 1419-1428 (2012)

23. Khan, A.A., Tammer, C., Zalinescu, C.: Regularization of quasi-variational inequalities. Optimization 64, 1703-1724 (2015)

24. Khan, A.A., Migórski, S., Sama, M.: Inverse problems for multi-valued quasi-variational inequalities and noncoercive variational inequalities with noisy data. Optimization 68, 1897-1931 (2019)

25. Kluge, R.: On some parameter determination problems and quasi-variational inequalities, in: Theory of Nonlinear Operators, vol. 6, pp. 129-139, Akademie-Verlag, Berlin (1978)

26. Li, X.W., Liu, Z.H.: Sensitivity analysis of optimal control problems described by differential hemivariational inequalities. SIAM J. Control Optim. 56, 3569-3597 (2018)

27. Liu, Z.H., Motreanu, D., Zeng, S.D.: On the well-posedness of differential mixed quasi-variational inequalities. Topol. Methods Nonlinear Anal. 51, 135-150 (2018)

28. Liu, Z.H.: Browder-Tikhonov regularization of non-coercive evolution hemivariational inequalities. Inverse Problems 21, 13-20 (2004)

29. Liu, Z.H.: Existence results for quasilinear parabolic hemivariational inequalities. J. Differential Equations 244, 1395-1409 (2008)

30. Liu, Z.H., Li, X.W., Motreanu, D.: Approximate controllability for nonlinear evolution hemivariational inequalities in Hilbert spaces. SIAM J. Control Optim. 53, 3228-3244 (2015)

31. Liu, Z.H., Zeng, B.: Optimal control of generalized quasi-variational hemivariational inequalities and its applications. Appl. Math. Optim. 72, 305-323 (2015)

32. Migórski, S. Khan, A. A., Zeng, S. D.: Inverse problems for nonlinear quasi-variational inequalities with an application to implicit obstacle problems of $p$-Laplacian type, Inverse Problems 35, ID 035004, $14 \mathrm{pp}$ (2019)

33. Migórski, S., Khan, A.A., Zeng, S.D.: Inverse problems for nonlinear quasi-hemivariational inequalities with application to mixed boundary value problems, Inverse Problems 36, ID 024008, 20 pp (2020)

34. Migórski, S., Dudek, S.: Evolutionary Oseen model for generalized Newtonian fluid with multivalued nonmonotone friction law. J. Math. Fluid Mech. 20, 1317-1333 (2018)

35. Migórski, S., Dudek, S.: A new class of variational-hemivariational inequalities for steady Oseen flow with unilateral and frictional type boundary conditions. Zeitschrift Angew. Math. Mech. 100, e201900112 (2020)

36. Migórski, S., Ochal, A.: Hemivariational inequalities for stationary Navier-Stokes equations. J. Math. Anal. Appl. 306, 197-217 (2005)

37. Migórski, S., Ochal, A., Sofonea, M.: Nonlinear Inclusions and Hemivariational Inequalities. Models and Analysis of Contact Problems, Advances in Mechanics and Mathematics 26, Springer, New York, (2013)

38. Monteiro, R.D.C., Svaiter, B.F.: Complexity of variants of Tseng'ss modified FB splitting and Korpelevich's methods for hemivariational inequalities with applications to saddle-point and convex optimization problems. SIAM J. Optim. 21, 1688-1720 (2011)

39. Naniewicz, Z., Panagiotopoulos, P.D.: Mathematical Theory of Hemivariational Inequalities and Applications. Dekker, New York (1995)

40. Panagiotopoulos, P.D.: Hemivariational Inequalities, Applications in Mechanics and Engineering, Springer-Verlag, Berlin, (1993)

41. Tang, G.J., Huang, N.J.: Existence theorems of the variational-hemivariational inequalities. J. Global Optim. 56, 605-622 (2013)

42. Xiao, Y.B., Huang, N.J.: Browder-Tikhonov regularization for a class of evolution second order hemivariational inequalities. J. Global Optim. 45, 371-388 (2009) 
43. Xiao, Y.B., Yang, X.M., Huang, N.J.: Some equivalence results for well-posedness of hemivariational inequalities. J. Global Optim. 61, 789-802 (2015)

44. Yao, J.C., Zheng, X.Y., Zhu.: Stable minimizers of $\varphi$-regular functions, SIAM J. Optim. 27, 1150-1170 (2017)

45. Zeidler, E.: Nonlinear Functional Analysis and its Applications II/B: Nonlinear Monotone Operators. Springer-Verlag, New York (1990)

Publisher's Note Springer Nature remains neutral with regard to jurisdictional claims in published maps and institutional affiliations.

\section{Authors and Affiliations}

\section{Shengda Zeng ${ }^{1,2} \cdot$ Dumitru Motreanu ${ }^{3,4} \cdot$ Akhtar A. Khan $^{5}$}

$凶 \quad$ Shengda Zeng

shengdazeng@gmail.com ; shdzeng@hotmail.com ; zengshengda@163.com

Dumitru Motreanu

motreanu@univ-perp.fr

Akhtar A. Khan

aaksma@rit.edu

1 Guangxi Colleges and Universities Key Laboratory of Complex System Optimization and Big Data Processing, Yulin Normal University, Yulin 537000, People's Republic of China

2 Faculty of Mathematics and Computer Science, Jagiellonian University in Krakow, ul. Lojasiewicza 6, 30348 Krakow, Poland

3 College of Science, Yulin Normal University, Yulin, Guangxi 537000, People's Republic of China

4 Département de Mathématiques, Université de Perpignan, 66860 Perpignan, France

5 School of Mathematical Sciences, Rochester Institute of Technology, Rochester, New York 14623, USA 